\title{
ON A VECTOR SPACE CONSTRUCTION BY HAUSDORFF ${ }^{1}$
}

\section{B. J. PETTIS}

Introduction. Some years ago Hausdorff, using Hamel bases, showed that any infinite dimensional real Banach space contained a second category linear subspace that was not complete under any equivalent norm [5]. It is shown below that a slight abstraction of his construction leads to the following: (A) a "multiple offender" example (Theorem 3), one that combines several pathological features which have been separately discussed in the literature; ${ }^{2}$ (B) the partitioning of any infinite dimensional second category real linear topological space into a continuum of pairwise disjoint linear manifolds each of which is everywhere dense in the space (a result comparable to the partitionings of certain linear topological spaces into disjoint everywhere dense convex sets by Tukey [15] and Klee [8]); (C) some miscellaneous observations, including the fact that in some recent topological group theorems certain hypotheses cannot be dropped.

To be precise concerning (A), we shall establish the following theorem, in which $E^{n}$ is $n$-dimensional real Euclidean vector space and $L_{c}$ represents, for any element $c$ of $E^{n}$, the set of rational multiples of $c$. Denoting complements by primes, a set $E$ in $E^{n}$ will be called $m$-thick if both $E$ and $E^{\prime}$ have zero interior measure; when $E$ is in a topological space $X$ it is $c$-thick if each of the sets $E$ and $E^{\prime}$ contains no Baire subsets of $X$ other than first category sets.

THEOREM 3. In $E^{n}$ there exists a subgroup $L$ that (i) is maximal with respect to being a proper subgroup closed under multiplication by rational scalars, (ii) has vacuous interior and is second category, everywhere dense, and non-Baire, (iii) is c-thick, (iv) is m-thick, (v) is nonmeasurable, (vi) is the set of zeroes of an open additive discontinuous map of $E^{n}$ onto the rationals, and (vii) is the image set of an open additive discontinuous projection defined on $E^{n}$. In addition, (viii) for any $c$ not in $L$ the function $(s, t) \rightarrow s+t$ maps $L \times L_{c}$ onto $E^{n}$ in a fashion that is additive, 1-1, and continuous but not bicontinuous.

For $n=1$ the existence of a subgroup satisfying (vi) is, of course,

Presented to the Society, September 7, 1951 ; received by the editors July 14, 1951 and, in revised form, July 15, 1956.

1 This paper was written under Contract N7-ONR-434, Office of Naval Research.

2 A discontinuous solution of the functional equation $f(x+y)=f(x)+f(y)$ has been effectively used by F. B. Jones [7] to provide a "multiple-valued" example in connection with connectivity properties. 
the classical result of Hamel [4]. An $m$-thick set has been called thick by Halmos [2] and saturated nonmeasurable by Halperin [3]; for $n=1$ the latter showed that (vi) implies (iv), and for $n=2$ the existence of an $m$-thick subgroup was established by Kodaira [9, 2, p. 267]. The proof below for arbitrary $n$ is taken from Halperin's paper. We are not aware of any subgroup of $E^{n}$ previously exhibited as explicitly being $c$-thick. The situation described in (viii) is well known; in the standard examples however (e.g., [13, p. 71]), it is the factor groups which are well behaved rather than the decomposed group.

Hausdorff's construction. Let $X$ be an additive Abelian group, with zero element $\theta$, having a fixed subsfield $\Omega=[\omega]$ in its ring of endomorphisms. A subgroup $L$ of $X$ is an $\Omega$-subgroup if $\omega(L) \subset L$ holds for every $\omega$ in $\Omega$; a function $\phi$ on $X$ to either $X$ or $\Omega$ is $\Omega$-homogeneous if $\phi(\omega(x))=\omega(\phi(x))$ for all $x$ and $\omega$, and is distributive if it is additive and $\Omega$-homogeneous.

Suppose that $X$ has infinite $\Omega$-dimension, i.e., that among its Hamel bases with respect to $\Omega$ there is at least one, $A$, which is infinite. Choosing $\left\{a_{i}\right\}$ to be a denumerable sequence of distinct elements in $A$, let $A_{n}$ consist of $A$ with the set $\left[a_{i}: i>n\right]$ deleted and let $L_{n}$ be the $\Omega$-subgroup generated by $A_{n}$. Clearly $X=\cup_{1}^{\infty} L_{n}$ and no $L_{n}$ is $X$. Now let $\sum_{j}, j=1,2, \cdots, r$, be a finite number of $\sigma$-ideals of subsets of $X$ no one of which contains $X$ as an element. For each $j$ there must be some $k_{j}$ such that $L_{k_{j}} \notin \sum_{j}$ : for otherwise $X=\cup_{1}^{\infty} L_{n}$ $\in \sum_{j}$; choosing $k=\max _{j} k_{j}$, it follows that, for every $j, L_{k} \supset L_{k_{j}}$ and hence $L_{k} \notin \sum_{j}$. Let $b=a_{k+1}$. Since $b \notin L_{k}$, there exists by the Hausdorff Maximality Principle a subset $L$ of $X$ that is maximal with respect to being an $\Omega$-subgroup containing $L_{k}$ but not containing $b$. By a standard argument in vector spaces it then follows that $L$ is a maximal proper $\Omega$-subgroup. Moreover, $L$ is clearly not in any $\sum_{j}$. This establishes the following:

THEOREM 1. If $X$ has infinite $\Omega$-dimension and is not an element of any of the $\sigma$-ideals $\sum_{j}, j=1,2, \cdots, r$, there exists a maximal proper $\Omega$-subgroup $L$ in $X$ that is not an element of any $\sum_{j}$.

For any $c$ in $X$ let $L_{c}$ be the $\Omega$-subgroup generated by $c$, i.e., $L_{c}$ $=[\omega(c): \omega \in \Omega]$.

Theorem 2. In addition to the hypotheses in Theorem 1, suppose $X$ has a topology such that $x+y$ and $-x$ are continuous in $x$ and $X$ is second category and connected. Then (1) there exists a maximal proper $\Omega$-subgroup $L$ that is not an element of any $\sum_{j}$ and which has properties 
(ii) and (iii) of Theorem 3. Moreover, for any fixed $c$ in the complement $L^{\prime}$ of $L$ we have the following: (2) there exists a distributive map of $X$ onto $\Omega$ that is discontinuous for all $T_{1}$-topologies in $\Omega$ and has $L$ for its set of zeroes; (3) if the topology in $X$ is $T_{1}$ there is a distributive open discontinuous projection of $X$ onto $L_{c}$ having $L$ for its zeroes, and the function $(s, t) \rightarrow s+t$ is an algebraic $\Omega$-isomorphism of $L \times L_{c}$ onto $X$ having its inverse discontinuous; (4) if the topology in $X$ is $T_{1}$ and $x-y$ is continuous in $(x, y)$, there is a distributive open discontinuous projection of $X$ onto $L$ having $L_{c}$ for its zeroes, and the map $(s, t) \rightarrow s+t$ is continuous but not bicontinuous.

Since $X$ is second category we may adjoin to the $\sum_{j}$ 's the $\sigma$-ideal of first category sets in $X$ and assert that the subgroup $L$ given by Theorem 1 is second category. Having $X$ connected, it then follows that the interior of $L$ is vacuous and the only Baire subsets of $X$ lying in $L$ are first category sets, since otherwise [12, Corollary 6] $L$ is an open and closed proper subgroup of $X$, contrary to $X$ being connected. Since $L$ is second category we can thereby conclude also that it is non-Baire. Moreover $L$ is everywhere dense since its closure, being a closed second category subgroup, is also open and so necessarily coincides with $X$. To complete the verification of (iii) of Theorem 3, let $I I(L)$ consist of those points in $X$ at each of which $L$ is second category. From the fact that $L$ is a second category subgroup it follows that $I I(L) \supset L$, and since $I I(L)$ is closed and $L$ is everywhere dense we have $I I(L)=X$. This shows [12, Lemma 2] that the complement $L^{\prime}$ of $L$ can contain no second category Baire subsets of $X$.

Let $c$ be fixed in $L^{\prime}$. Since $L$ is a maximal proper $\Omega$-subgroup, each $x$ in $X$ has a unique representation $x=\pi(x)+\omega_{x}(c)$ with $\pi(x) \in L$ and $\omega_{x}(c) \in L_{c}$; moreover, $\pi$ is a distributive projection of $X$ onto $L$, $\omega_{x}(c)$ is a distributive projection of $X$ onto $L_{c}, \omega_{x}$ is distributive on $X$ onto $\Omega$, and $L$ is the set of zeroes of $\omega_{x}$ and of $\omega_{x}(c)$ and $L_{c}$ is that of $\pi$. Being projections, $\pi(x)$ and $\omega_{x}(c)$ are necessarily open maps onto $L$ and $L_{c}$ respectively. Since $L$ is not closed $\omega_{x}$ is discontinuous for any $T_{1}$-topology in $\Omega$; similarly, $\omega_{x}(c)$ is discontinuous if the topology in $X$ is $T_{1}$. If we define $\phi$ on $X$ to $L \times L_{c}$ by $\phi(x)=\left(\pi(x), \omega_{x}(c)\right)$, it can be seen that $\phi$ is an $\Omega$-isomorphism onto, that $\phi^{-1}$ is given by $\phi^{-1}(s, t)$ $=s+t$, and that $\phi$ is not continuous since $\omega_{x}(c)$ is not. If $x-y$ is continuous in $(x, y)$ clearly $\phi^{-1}$ is continuous and, since $\omega_{x}(c)=x-\pi(x)$, $\pi$ is not continuous. This completes the proof of Theorem 2 .

To establish Theorem 3 let $X$ be $E^{n}$, take $\Omega$ to consist of all multiplications by rational scalars, and let the sets of measure zero form one of the $\sum_{j}$ 's. The subgroup $L$ given by Theorem 2 clearly satisfies 
statements (i)-(iii) of Theorem 3 and has positive exterior measure; and from (3) and (4) of Theorem 2 properties (vi)-(viii) result for $L$. Concerning (iv), we first note that $L$ has zero interior measure, for it is a subgroup with vacuous interior and hence can contain no measurable set of positive measure [2, Theorems 62.B and 61.A]; this in turn obviously implies (v). Showing that $L^{\prime}$ has null interior measure is more difficult. ${ }^{3}$ Let $I$ be any nonnull open interval in $E^{n}$ and denote exterior and interior measure by $m_{\boldsymbol{e}}$ and $m_{i}$. For any $x$ in $L$ we have $m_{\bullet}(L \cap(x+I))=m_{\bullet}(x+[(-x+L) \cap I])=m_{\bullet}((-x+L) \cap I)$ $=m_{0}(L \cap I)$; and since $L$ is everywhere dense in $E^{n}$ we can, for any $x$ in $E^{n}$, choose $\left\{x_{n}\right\} \subset L$ such that $\lim x_{n}=x$ and obtain $m_{e}(L \cap(x+I))$ $=\lim _{n} m_{e}\left(L \cap\left(x_{n}+I\right)\right)=\lim m_{e}(L \cap I)$. Thus $\left(^{*}\right) m_{e}(L \cap(x+I))$ $=m_{\theta}(L \cap I)$ for any $x$ in $E^{n}$. From $(*)$ it follows in particular that for no $I$ do we have $m_{e}(L \cap I)=0$, for by the Lindelöf theorem a countable number $\left\{I_{k}\right\}$ of translations of $I$ cover $E^{n}$ and hence $0<m_{e}(L)$ $=m_{e}\left(U_{k}\left(L \cap I_{k}\right)\right) \leqq \sum_{1}^{\infty} m_{\theta}\left(L \cap I_{k}\right)=\sum_{1}^{\infty} m_{e}(L \cap I)$. Let $I_{+}=E^{n}[x:$ $0<x(i)<1, i=1,2, \cdots, n]$ be the open positive unit cube; then $m_{e}\left(L \cap I_{+}\right)=\delta=\delta m\left(I_{+}\right)$where $\delta$ is positive. For any rational number $\rho$ we have $L \cap \rho I_{+}=\rho L \cap \rho I_{+}=\rho\left(L \cap I_{+}\right)$and so $m_{e}\left(L \cap \rho I_{+}\right)$ $=\rho m_{e}\left(L \cap I_{+}\right)=\delta m\left(\rho I_{+}\right)$. From this and from $\left(^{*}\right)$ we obtain $m_{\bullet}(L \cap I)$ $=\delta m(I)$ for any open cube $I$ having rational edge length. Now suppose that $M$ is some measurable set lying in $L^{\prime}$ and having positive measure. From the density theorem $[14$, p. 117] it follows that there is at least one point $x_{0}$ in $M$ such that $\lim m(M \cap I) / m(I)=1$ as $I$ varies over cubes with center at $x_{0}$ and as $m(I) \rightarrow 0$. We may then choose an open rational cube $I_{\delta}$ such that $m\left(M \cap I_{\delta}\right) / m\left(I_{\delta}\right)>1-\delta$; but then $m\left(I_{\delta}\right)=m_{\theta}\left(L \cap I_{\delta}\right)+m_{i}\left(L^{\prime} \cap I_{\delta}\right) \geqq m_{\delta}\left(L \cap I_{\delta}\right)+m\left(M \cap I_{\delta}\right)$ $>\delta m\left(I_{\delta}\right)+(1-\delta) m\left(I_{\delta}\right)=m\left(I_{\delta}\right)$. This confirms Theorem 3 .

Everywhere dense linear manifolds. The cosets of $L$ in Theorem 2 clearly are pairwise disjoint, have the same cardinal number as $\Omega$, and are each everywhere dense in $X$. This gives us the following:

TheOREM 4. Any second category infinite dimensional real linear topological space $X$ is the union of $2 \aleph_{0}$ pairwise disjoint linear manifolds each of which is everywhere dense in $X$.

Since a linear manifold is convex this compares with results of Tukey [15] and Klee [8]. In this connection we remark that the standard proof of the well known fact that any infinite dimensional real linear normed space $X$ is the domain of a distributive discon-

3 As remarked earlier, the proof is a slight paraphrase of one by Halperin; it can be used to extend his theorem from $E^{1}$ to $E^{n}$. 
tinuous real function actually shows that such a function $f$ exists if $X$ is a real linear topological space containing a bounded infinite set of linearly independent elements. Letting $Z$ be the zeroes of $f, Z$ is a maximal proper linear subspace and cannot be closed since $f$ is not continuous. This implies that the closure of $Z$ is $X$, and hence that $Z$ is everywhere dense. Considering the cosets of $Z$ it follows that the conclusion of Theorem 4 holds for such an $X$ and hence for any infinite dimensional locally bounded [6] real linear topological space and therefore for any infinite dimensional real linear normed space. In particular, it holds for $L_{p}$ and $l_{p}$ when $0<p<1$.

Concluding remarks. (1) In any real linear space $X$ consider the set $[\nu]$ of all non-negative functions $\nu$ on $X$ which are positive homogeneous, symmetric, and vanish at $\theta$. Defining the neighborhoods of $\theta$ to be all sets of the form $X[x: \nu(x)<1], \nu \in[\nu]$, and taking the neighborhoods in $X$ to be all translations of these, $X$ is a topological space in which $x+y$ and $-x$ are continuous in $x, \lambda x$ is continuous in real $\lambda$ for each $x$, and every real distributive function on $X$ is continuous. If $X$ is infinite dimensional it follows from Theorem 2 that in this so-called "radial topology" $X$ is first category.

(2) The subgroup and the homomorphisms described in Theorem 3 show that in many of the results given in $[10 ; 11 ; 12]$ certain assumptions cannot be dropped, e.g., that a domain or range space is right complete, or that a homomorphism kernel is closed, or that a set under consideration is a Baire set. For example, as Klee has pointed out [16, p. 450] McShane's extension [10] of a theorem by Eberlein, to the effect that any second category Baire set which is midpoint convex has a non-null interior, clearly fails when "Baire" is omitted.

(3) V. L. Klee has pointed out to the author that Theorem 2 and a well known result of Sierpinski's [17] on absolute $G_{\delta}$-sets show that Hausdorff's original statement holds in the following form: in any infinite dimensional second category metrizable linear topological space there always exists a second category linear subspace $L$ that is homeomorphic to no complete metric space. He has also remarked that Erdös noted in a letter that, by using a construction very similar to Hausdorff's, every infinite dimensional linear space is the union of countably many maximal proper subspaces.

\section{REFERENCES}

1. S. Banach, Theorie des operations lineaires, Warsaw, 1932.

2. P. R. Halmos, Measure theory, New York, Van Nostrand, 1950. 
3. I. Halperin, Non-measurable sets and the equation $f(x+y)=f(x)+f(y)$, Proc. Amer. Math. Soc. vol. 2 (1951) pp. 221-224.

4. G. Hamel, Eine Basis aller Zählen und die unstetigen Lösungen der Funktionalgleichung: $f(x+y)=f(x)+f(y)$, Math. Ann. vol. 60 (1905) pp. 459-462.

5. F. Hausdorff, Zur Theorie der linearen metrischen Räume, J. Reine Angew. Math. vol. 167 (1932) pp. 294-311.

6. D. H. Hyers, $A$ note on linear topological spaces, Bull. Amer. Math. Soc. vol. 44 (1938) pp. 76-80.

7. F. B. Jones, Connected and disconnected plane sets and the functional equation $f(x)+f(y)=f(x+y)$, Bull. Amer. Math. Soc. vol. 48 (1942) pp. 115-120.

8. V. L. Klee, Jr., Dense convex sets, Duke Math. J. 16 (1949) pp. 351-354.

9. K. Kodaira, Über die Beziehung zwischen den Massen und Topologien in einer Gruppe, Proceedings Physico-Mathematical Society of Japan vol. 23 (1941) pp. 67119.

10. E. J. McShane, Images of sets satisfying the condition of Baire, Ann. of Math. vol. 51 (1950) pp. 380-386.

11. B. J. Pettis, On continuity and openness of homomorphisms in topological groups, Ann. of Math. vol. 52 (1950) pp. 293-308.

12. - Remarks on a theorem of E. J. McShane, Proc. Amer. Math. Soc. vol. 2 (1951) pp. 166-171.

13. L. Pontrjagin, Topological groups, Princeton, Princeton University Press, 1946.

14. S. Saks, Theory of the integral, Warsaw, 1937.

15. J. W. Tukey, Some notes on the separation of convex sets, Portugaliae Mathematica vol. 3 (1942) pp. 95-102.

16. V. L. Klee, Jr., On convex sets in linear spaces, Duke Math. J. vol. 18 (1951) pp. 443-466.

17. W. Sierpinski, Sur les ensembles complets d'un espace (D), Fund. Math. vol. 11 (1928) pp. 203-205.

\section{Tulane University}

